\title{
Towards next-to-leading order corrections to the heavy quark potential in the effective string theory
}

\author{
Sungmin Hwang ${ }^{1,2, a}$ \\ ${ }^{1}$ Physik-Department I, Technische Universität München, T30f \\ ${ }^{2}$ Max-Planck-Institut für Physik (Werner-Heisenberg-Institut), München
}

\begin{abstract}
We present our calculation of the non-relativistic corrections to the heavy quark-antiquark potential up to leading and next-to-leading order (NLO) via the effective string theory (EST). Full systematics of effective field theory (EFT) are discussed in order for including the NLO contribution that arises in the EST. We also show how the number of dimensionful parameters arising from the EST are reduced by the constraints between the Wilson coefficients from non-relativistic EFTs for QCD.
\end{abstract}

\section{Introduction}

Confinement in quantum chromodynamics (QCD) is one of the greatest challenges in modern theoretical particle physics community [1]. It raises an issue that the conventional perturbative approach in $S U(3)$ gauge theory, i.e., the perturbative QCD (pQCD), is no longer a feasible framework for understanding the color interactions amongst particles around the strong scale $\Lambda_{Q C D}$ (or at the pion mass scale $m_{\pi}$ which is about $200 \mathrm{MeV}$ ), although it has proven to be successful in the higher energy processes; only the composite forms of particles like various kind of mesons and baryons are detected in the form of jets instead of isolated quarks/antiquarks or gluons, in the experiments.

Despite the absence of the complete perturbative framework for describing transitions from deconfining to confining phase, there have been a wide variety of low-energy EFTs for QCD concerning the physical processes of one's interests, such as chiral perturbation theory $(\chi P T)$ for light mesons $(\pi, \sigma, \rho$, etc), in which the mesons are the dynamical degrees of freedom, and the lattice gauge theory valid at the non-perturbative regime. Besides the great success of these low-energy EFTs for QCD, another type of physical process concerning the heavy particles, such as charm and bottom quarks, is not to be overlooked because many important particle interactions are processed through either productions or annihilations of the heavy mesons. Their dynamics are non-relativistic at the low-energy regime due to its heavy mass, and it is well described by the series of low-energy EFTs, such as heavy quark effective theory (HQET) for the heavy quark and light antiquark states, non-relativistic QCD (NRQCD) for the heavy quark and heavy antiquark states, and potential NRQCD (pNRQCD) for the heavy quark-antiquark bound states. Since these EFTs are valid well above the confinement scale, another kind of the EFT framework concerning

a e-mail: sungmin.hwang@tum.de 
the heavy quark-antiquark states for the energy scale below $\Lambda_{Q C D}$ is also required to understand its long-distance behavior.

Since the realization of confinement in QCD, Nambu has proposed that the bound states can be described by the flux tube model [2], in which the quark and the antiquark pair is connected by gluon lines, whose tension increases as these particles are separated at the long distance limit $\left(L>\Lambda_{Q C D}^{-1}\right)$, thereafter its flux forms in the shape of a tube. In this formulation, the heavy quark-antiquark pair is treated as a static object, and the gluodynamics is described by the vibrating string which connects the pair. Since the two ends of the string are fixed at the position of the heavy quark and antiquark, respectively, only the transversal modes are counted as the degrees of freedom. Kogut and Parisi, after several years, developed this idea further so that the spin-spin interaction part of the potential energy between the heavy quark-antiquark pair was explicitly shown [3].

In this fashion, significant progress has been made during the last decades. Via the so-called matching calculation, potential terms of the heavy quark-antiquark pair were shown to be equivalent to the Wilson loop and the gauge fields insertions therein, at the large time limit $[4,5]$, and based on this premise as well as on the hypothesis of the Wilson loops-string action equivalence, a few of the potential terms were directly computed through the string picture [6]. Recently, Brambilla et. al. have analyzed all of the heavy quark potentials via the EST up to the leading order, from which it was compared to the lattice simulations/calculations in order to constrain some of the parameters arising from the EFT framework [7]. As it was pointed out in this paper, however, this leading order calculation is not fully inclusive because some of its NLO contributions would contain terms which are in the same order of the expansion parameter; in other words, NLO terms might alter the leading order coefficients of the potentials, so the impact of the NLO contribution is not to be underestimated. Therefore, it is necessary for us to implement the proper EFT systematics in the string picture, such that not only the higher order suppression terms are fully understood but all of the missing terms from the LO calculation are also acquired in order to make the better fit to the lattice data.

In the direction to clarifying these issues, this proceeding paper is organized as the following: in Sec. 2, we introduce NRQCD for a non-relativistic system of the heavy quark and heavy antiquark pair, its Lagrangian and Wilson coefficients, as well as the framework of pNRQCD, the lower-energy counterpart of NRQCD for its bound states. Through the introduction of these low-energy EFTs for QCD, equivalence between the Wilson loop and gauge fields insertions therein from NRQCD and potential terms from pNRQCD at the large time limit are established. In Sec. 3, we introduce the framework of the EST in which the hierarchy of scales, its degrees of freedom and symmetries of the theory are discussed. Based on the equivalence between the string partition function and Wilson loop expectation value at large time limit, our analysis of the heavy quark potentials is presented. In Sec. 4, we show how the full EFT systematics of the EST can be implemented, from which we show the schematic calculation to the NLO contributions to the heavy quark potentials. Finally, we give its summary and outlook in Sec. 5.

\section{Non-relativistic EFTs for QCD}

For non-relativistic dynamics between heavy quarks and antiquarks, a hierarchy of scale arises: $M \gg$ $M v \gg \Lambda_{Q C D}$ in which $M$ denotes the mass of the heavy quark/antiquark and $v$ is the relative velocity in between (thus, $M v$ denotes the relative momentum). The NRQCD Lagrangian, up to the bilinear 
sector in heavy fields, is then obtained by integrating out the hard scale $M[8,9]$

$$
\begin{aligned}
\mathcal{L}_{N R Q C D} & =\psi^{\dagger}\left\{i D_{0}+c_{1} \frac{\mathbf{D}^{2}}{2 M}+c_{2} \frac{\mathbf{D}^{4}}{8 M^{3}}+c_{F} g \frac{\boldsymbol{\sigma} \cdot \mathbf{B}}{2 M}+c_{D} g \frac{[\mathbf{D} \cdot \mathbf{E}]}{8 M^{2}}+i c_{s} g \frac{\boldsymbol{\sigma} \cdot[\mathbf{D} \times, \mathbf{E}]}{8 M^{2}}+O\left(M^{-3}\right)\right\} \psi \\
& +\chi^{\dagger}\left\{i D_{0}-c_{1} \frac{\mathbf{D}^{2}}{2 M}-c_{2} \frac{\mathbf{D}^{4}}{8 M^{3}}-c_{F} g \frac{\boldsymbol{\sigma} \cdot \mathbf{B}}{2 M}+c_{D} g \frac{[\mathbf{D} \cdot \mathbf{E}]}{8 M^{2}}+i c_{s} g \frac{\boldsymbol{\sigma} \cdot[\mathbf{D} \times, \mathbf{E}]}{8 M^{2}}+O\left(M^{-3}\right)\right\} \chi \\
& -\frac{1}{4} F_{\mu \nu}^{a} F^{\mu v, a}+\mathcal{L}_{\text {light }}
\end{aligned}
$$

in which $\psi$ and $\chi$ are Pauli spinor fields which annihilates the heavy quark and creates the heavy antiquark, respectively, $F^{2}$ is the field strength term for the $S U(3)$ gauge symmetry (soft gluon fields), and $\mathcal{L}_{\text {light }}$ is the same Dirac Lagrangian for light quarks; in addition $c_{1}, c_{2}, c_{F}, c_{D}$ and $c_{S}$ are the Wilson coefficients which contain the information from its underlying theory, i.e., pQCD. This EFT is useful for calculating productions and annihilations of the heavy quark-heavy antiquark states, but it is not easy to analyze the transitions or the mass splittings of the heavy quarkonium states with this framework.

An even lower-energy counterpart for NRQCD, namely potential NRQCD (pNRQCD), is able to give a better description for such processes. Its hierarchy of scale is manifested like $M \gg M v \gg M v^{2}$, in which $M v^{2}$ denotes the relative kinetic energy in between. Thus, the Lagrangian of pNRQCD is obtained after integrating out the soft scale $M v$, which is equivalent to the inverse distance between the heavy quark and antiquark pair, $1 / r$; we utilize the multipole expansion in addition to the $1 / M$ expansion in NRQCD. Its degrees of freedom are then consist of the color singlet $S(r, R, t)$ and octet $O^{a}(r, R, t)$ fields, which have a different coordinate dependence than in NRQCD: real time $t$, relative distance $\mathbf{x}_{1}-\mathbf{x}_{2}=\mathbf{r}$, and the center of mass coordinate $\left(\mathbf{x}_{1}+\mathbf{x}_{2}\right) / 2=\mathbf{R} ; \mathbf{x}_{1}$ is the position of the heavy quark and $\mathbf{x}_{2}$ is the position of the heavy antiquark. Dirac spinor fields for the light quarks and ultra-soft gluons for the $S U(3)$ gauge symmetry are included as well. Heuristic form of the pNRQCD Lagrangian (up to the bilinear sector in singlet and octet fields) is expressed by [10]

$$
\begin{aligned}
\mathcal{L}_{p N R Q C D}= & \operatorname{Tr}\left[S^{\dagger}\left(i \partial_{0}-V_{S}(r)+\ldots\right) S+O^{\dagger}\left(i D_{0}-V_{O}(r)+\ldots\right) O\right]+g V_{A}(r) \operatorname{Tr}\left[O^{\dagger} \mathbf{r} \cdot \mathbf{E} S+S^{\dagger} \mathbf{r} \cdot \mathbf{E} O\right] \\
& +g \frac{V_{B}(r)}{2} \operatorname{Tr}\left[O^{\dagger} \mathbf{r} \cdot \mathbf{E} O+O^{\dagger} O \mathbf{r} \cdot \mathbf{E}\right]-\frac{1}{4} F_{\mu \nu}^{a} F^{\mu v, a}+\mathcal{L}_{\text {Light }} .
\end{aligned}
$$

It is important to note that $V$ 's are the Wilson coefficients, various forms of the heavy quark potentials. As we analyze the singlet part of the potentials with including up to the second order in $1 / M$ expansion, its heuristic expression is given by the following

$$
\begin{aligned}
V(r)=V^{(0)}(r)+\frac{2}{M} V^{(1,0)}(r) & +\frac{1}{M^{2}}\left\{\left[2 \frac{V_{\mathbf{L}^{2}}^{(2,0)}(r)}{r^{2}}+\frac{V_{\mathbf{L}^{2}}^{(1,1)}(r)}{r^{2}}\right] \mathbf{L}^{2}+\left[V_{L S}^{(2,0)}(r)+V_{L_{2} S_{1}}^{(1,1)}(r)\right] \mathbf{L} \cdot \mathbf{S}\right. \\
& \left.+V_{S^{2}}^{(1,1)}(r)\left(\frac{\mathbf{S}^{2}}{2}-\frac{3}{4}\right)+V_{\mathbf{S}_{12}}^{(1,1)}(r) \mathbf{S}_{12}(\hat{\mathbf{r}})+2 V_{r}^{(2,0)}(r)+V_{r}^{(1,1)}(r)\right\},
\end{aligned}
$$

in which the tensor component of the spin-spin interaction part is defined as: $\mathbf{S}_{12}(\hat{\mathbf{r}}) \equiv 3 \hat{\mathbf{r}} \cdot \sigma_{1} \hat{\mathbf{r}}$. $\sigma_{2}-\sigma_{1} \cdot \sigma_{2}$. Clarification to the notation used here is in order: the superscript denotes the order of expansions, in which the first entry of the parenthesis represents the order in $1 / M_{1}$ expansion $\left(M_{1}\right.$ being the mass of the heavy quark), and the second entry is the order in $1 / M_{2}$ expansion $\left(M_{2}\right.$ is the mass of the heavy antiquark). Also, $\mathbf{S}_{1}=\sigma_{1} / 2$ is the spin projection for the heavy quark and $\mathbf{S}_{\mathbf{2}}=\sigma_{\mathbf{2}} / 2$ is for the heavy antiquark. These Wilson coefficients are determined by the matching 
procedure with its UV theory, NRQCD in this case. Matching is performed in a way that we first calculate the Green's function for the quark-antiquark pair in NRQCD and compare it to the Green's function from the singlet sector in pNRQCD at the static limit. At the leading order in the matching, we find an important relation between the static singlet potential and the Wilson loop expectation value at large time

$$
V^{(0)}(r)=\lim _{T \rightarrow \infty} \frac{i}{T} \ln \left\langle W_{\square}\right\rangle
$$

in which $W_{\square}$ is the rectangular Wilson loop which is defined by

$$
W_{\square} \equiv P \exp \left\{-i g \oint_{r \times T} d x^{\mu} A_{\mu}(z)\right\}
$$

where $P$ stands for the path ordering of the color matrices and the angular bracket denotes its average value over the Yang-Mills action [4]. The first order corrections to the static singlet potentials are related to the gauge fields insertion to the Wilson loop expectation value as the matching proceeds towards the subleading contributions up to the first order in $1 / M_{1}$ or $1 / M_{2}$ [4]

$$
\begin{aligned}
V^{(1,0)}(r) & =-\frac{1}{2} \lim _{T \rightarrow \infty} \int_{0}^{T} d t t\left\langle\left\langle g \mathbf{E}_{1}(t) \cdot g \mathbf{E}_{1}(0)\right\rangle\right\rangle_{c} \\
& =V^{(0,1)}(r) .
\end{aligned}
$$

Here the subscript on the gauge field denotes $\mathbf{E}_{1}(t) \equiv \mathbf{E}(t, r / 2)$ (so naturally $\mathbf{E}_{2}(t) \equiv \mathbf{E}(t,-r / 2)$ ), the double angular bracket is defined $\langle\langle\ldots\rangle\rangle \equiv\left\langle\ldots W_{\square}\right\rangle /\left\langle W_{\square}\right\rangle$, and the subscript $c$ represents the connected part of the gauge fields correlator, whose definition is given by

$$
\left\langle\left\langle O_{1}\left(t_{1}\right) O_{2}\left(t_{2}\right)\right\rangle\right\rangle_{c}=\left\langle\left\langle O_{1}\left(t_{1}\right) O_{2}\left(t_{2}\right)\right\rangle\right\rangle-\left\langle\left\langle O_{1}\left(t_{1}\right)\right\rangle\right\rangle\left\langle\left\langle O_{2}\left(t_{2}\right)\right\rangle\right\rangle, \text { for } t_{1} \geq t_{2},
$$

with $O_{i}\left(t_{i}\right)$ being a generic gauge field inserted into the expectation value (it would be either a chromoelectric or a chromomagnetic field in the calculations to be followed). The last line of Eq. (6) is due to the invariance under the exchange between the heavy quark mass and the heavy antiquark mass as well as to the charge conjugation. Second order corrections are classified into two categories, the first of which is the spin-independent part $V_{S I}^{(2,0)}+V_{S I}^{(1,1)}+V_{S I}^{(0,2)}$, and the second category is the spindependent contributions, $V_{S D}^{(2,0)}+V_{S D}^{(1,1)}+V_{S D}^{(0,2)}$; the superscripts for the expansion scheme was already introduced before. First term of the spin-independent contribution includes the following potentials, which are related to the multiple gauge fields insertion to the Wilson loop expectation value as

$$
\begin{aligned}
V_{\mathbf{p}^{2}}^{(2,0)}(r) & =\frac{i}{2} \hat{\mathbf{r}}^{\mathbf{i}} \mathbf{\mathbf { r } ^ { \mathbf { j } }} \int_{0}^{\infty} d t t^{2}\left\langle\left\langle g \mathbf{E}_{1}^{i}(t) g \mathbf{E}_{1}^{j}(0)\right\rangle\right\rangle_{c} \\
V_{\mathbf{L}^{2}}^{(2,0)}(r) & =\frac{i}{4}\left(\delta^{i j}-3 \hat{\mathbf{r}}^{\mathbf{i}} \hat{\mathbf{r}}^{\mathbf{j}}\right) \int_{0}^{\infty} d t t^{2}\left\langle\left\langle g \mathbf{E}_{1}^{i}(t) g \mathbf{E}_{1}^{j}(0)\right\rangle\right\rangle_{c} \\
V_{r}^{(2,0)}(r) & =\frac{\pi C_{f} \alpha_{s} c_{D}^{(1)^{\prime}}}{2} \delta^{(3)}(\mathbf{r})-\frac{i\left(c_{F}^{(1)}\right)^{2}}{4} \int_{0}^{\infty} d t\left\langle\left\langle g \mathbf{B}_{1}(t) \cdot g \mathbf{B}_{1}(0)\right\rangle\right\rangle_{c}+\ldots
\end{aligned}
$$

for which the normal vector in the radial direction is parametrized by $\hat{\mathbf{r}}=(0,0,1)$, and the ellipsis on the $V_{r}^{(2,0)}(r)$ contains three and four chromoelectric fields insertion to the Wilson loop expectation value. In addition, $V_{S I}^{(2,0)}=V_{S I}^{(0,2)}$ due to the exchange of masses and charge conjugation. $V_{S I}^{(1,1)}$ part is similar to these expressions, which are found in [5]. Spin-dependent part contains the following term

$$
V_{L S}^{(2,0)}(r)=-\frac{c_{F}^{(1)}}{r^{2}} i \mathbf{r} \cdot \int_{0}^{\infty} d t t\left\langle\left\langle g \mathbf{B}_{1}(t) \times g \mathbf{E}_{1}(0)\right\rangle+\frac{c_{S}^{(1)}}{2 r^{2}} \mathbf{r} \cdot\left(\nabla_{r} V^{(0)}\right)\right.
$$


and the remaining terms like $V_{L_{2} S_{1}}^{(1,1)}(r), V_{S^{2}}^{(1,1)}(r)$, and $V_{\mathbf{S}_{12}}^{(1,1)}(r)$ are also found in [5]. An important question one can raise at this point is how to investigate their analytic behavior at the long-distance regime. It has been addressed with a non-perturbative method like lattice QCD in order for obtaining the expressions for gauge fields insertions, but 3- and 4-gauge fields insertions (which are found in $V_{r}$ potentials) are not easily obtained from this method. Thus, our approach to address this question is by using the effective string theory (EST), which is a suitable EFT for the heavy quark-antiquark bound states at the long-distance limit, and comparing it to the lattice calculations/simulations in order to constrain several parameters arising within this EFT framework. We will present more detailed discussion about them in the next section.

\section{Wilson loops and the effective string theory (EST)}

A key hypothesis that we start with for connecting the low-energy EFTs for QCD to the EST is the Wilson loop-string action equivalence [11]

$$
\lim _{T \rightarrow \infty}\left\langle W_{\square}\right\rangle=Z \int \mathcal{D} \xi^{1} \mathcal{D} \xi^{2} e^{i S_{\text {string }}\left(\xi^{1}, \xi^{2}\right)}
$$

where $\xi^{i}(i=1,2)$ are the transversal vibration modes of a string which connects the static heavy quark and the heavy antiquark; the string originates from the gluodynamics. As the heavy quark is located at $(0,0, r / 2)$ and the heavy antiquark is at $(0,0,-r / 2)$, vibrational modes of the string (or string coordinates) $\xi^{i}$ are along the $x, y$ axes. $Z$ in Eq. (12) is a constant originating from the normalization of the string partition function, and $S_{\text {string }}$ is the action of the EST, whose construction is explained in the next paragraph.

First of all, its hierarchy of scale is to be observed. It was mentioned in the previous section that this low-energy EFT is valid at the long-distance limit, which is manifested by $r \Lambda_{Q C D} \gg 1$; i.e., the energy scale, in the order of $1 / r$, is much smaller than the confinement scale in QCD. Second condition was implicitly mentioned in the previous paragraph, such that two ends of the string coordinate are fixed at the position of the heavy quark and the heavy antiquark, which means that only the $x$ and $y$ components of the string vibration are dynamical degrees of freedom $\left(\xi^{1}, \xi^{2}\right)$. Then the power counting scheme is clear from these conditions: $\partial_{z, t} \sim 1 / r, \xi^{l} \sim 1 / \Lambda_{Q C D}$ (i.e., $\left(\partial_{a} \xi^{l} \sim\left(r \Lambda_{Q C D}\right)^{-1}\right.$; $a \in\{0,3\}, l \in\{1,2\})$. Starting from the Nambu-Goto action, the action for a relativistic string in the Minkowski spacetime valid at all energy level, its long-distance approximation in 4-dimensional spacetime with fixing the gauge (static gauge in this context) is given by

$$
\begin{aligned}
S_{\text {string }} & =-\sigma \int d t d z \sqrt{\operatorname{det}\left(\eta_{a b}+\partial_{a} \xi^{l} \partial_{b} \xi^{l}\right)} \\
& =-\sigma \int d t d z\left(1-\frac{1}{2} \partial_{a} \xi^{l} \partial^{a} \xi^{l}+\ldots\right)
\end{aligned}
$$

in which $\sigma$ is the string tension ( $\sim \Lambda_{Q C D}^{2}$, to be determined by lattice calculations), and the ellipsis includes higher order terms in the derivative expansion; it is clear that the first term inside the parenthesis gives rise to the static potential $V^{(0)}(r) \approx \sigma r$. For the subleading parts of the heavy quark potentials from QCD side, let us include the first non-trivial part of this action, which is naturally the truncation up to $\left(r \Lambda_{Q C D}\right)^{-2}$. We find, by solving the equations of motion from the Gaussian action in $\xi$, its Green's function in the Euclidean spacetime $(t \rightarrow-i t)$ to be [6]

$$
\left\langle\xi^{l}(-i t, z) \xi^{m}\left(-i t^{\prime}, z^{\prime}\right)\right\rangle=\frac{\delta^{l m}}{4 \pi \sigma} \ln \left(\frac{\cosh \left[\left(t-t^{\prime}\right) \pi / r\right]+\cos \left[\left(z+z^{\prime}\right) \pi / r\right]}{\cosh \left[\left(t-t^{\prime}\right) \pi / r\right]-\cos \left[\left(z-z^{\prime}\right) \pi / r\right]}\right) .
$$


Our next task is to determine a mapping from the gauge field insertion into the Wilson loop expectation value to the string dynamics. This mapping becomes more concrete as the symmetries of the physical system is realized, such as the rotational symmetry with respect to the $z$-axis, the reflection symmetry with respect to the $x z$-plane, invariance under $C P$ transformation as well as the time-reversal. Lastly, the mass dimension and the order of truncation, $\left(r \Lambda_{Q C D}\right)^{-2}$ are to be matched as well. All these conditions constrain the mapping as the following:

$$
\begin{aligned}
&\left.\left\langle\ldots \mathbf{E}_{1}^{l}(t) \ldots\right\rangle\right\rangle=\left\langle\ldots \Lambda^{2} \partial_{z} \xi^{l}(t, r / 2) \ldots\right\rangle \\
&\left.\left\langle\ldots \mathbf{E}_{2}^{l}(t) \ldots\right\rangle\right\rangle=\left\langle\ldots \Lambda^{2} \partial_{z} \xi^{l}(t,-r / 2) \ldots\right\rangle \\
&\left\langle\left\langle\ldots \mathbf{B}_{1}^{l}(t) \ldots\right\rangle\right\rangle=\left\langle\ldots \Lambda^{\prime} \epsilon^{l m} \partial_{t} \partial_{z} \xi^{m}(t, r / 2) \ldots\right\rangle \\
&\left\langle\left\langle\ldots \mathbf{B}_{2}^{l}(t) \ldots\right\rangle\right\rangle=\left\langle\ldots-\Lambda^{\prime} \epsilon^{l m} \partial_{t} \partial_{z} \xi^{m}(t,-r / 2) \ldots\right\rangle \\
&\left.\left\langle\ldots \mathbf{E}_{1}^{3}(t) \ldots\right\rangle\right\rangle=\left\langle\ldots \Lambda^{\prime \prime 2} \ldots\right\rangle \\
&\left.\left\langle\ldots \mathbf{E}_{2}^{3}(t) \ldots\right\rangle\right\rangle=\left\langle\ldots \Lambda^{\prime \prime 2} \ldots\right\rangle \\
&\left\langle\left\langle\ldots \mathbf{B}_{1}^{3}(t) \ldots\right\rangle\right\rangle=\left\langle\ldots \Lambda^{\prime \prime \prime} \epsilon^{l m} \partial_{t} \partial_{z} \xi^{l}(t, r / 2) \partial_{z} \xi^{m}(t, r / 2) \ldots\right\rangle \\
&\left.\left\langle\ldots \ldots \mathbf{B}_{2}^{3}(t) \ldots\right\rangle\right\rangle=\left\langle\ldots-\Lambda^{\prime \prime \prime} \epsilon^{l m} \partial_{t} \partial_{z} \xi^{l}(t,-r / 2) \partial_{z} \xi^{m}(t,-r / 2) \ldots\right\rangle
\end{aligned}
$$

in which $\Lambda, \Lambda^{\prime}, \Lambda^{\prime \prime}, \Lambda^{\prime \prime \prime}$ are dimensionful parameters in the order of $\Lambda_{Q C D}$, and the ellipsis on the left and right hand sides of the equation represents some additional gauge fields insertion and string fields, respectively [7]. One can first verify the equivalence for the chromoelectric fields purely from dimensional and symmetrical reasons, and the equivalence for the chromomagnetic fields are derived by the electromagnetic duality. With Eq. (14), (15) and the potentials from the previous section given, we are now ready to investigate the analytic behavior of the potentials. As a simple example, 2-chromoelectric field insertion is computed as

$$
\begin{aligned}
\left\langle\left\langle\mathbf{E}_{1}^{i}(-i t) \mathbf{E}_{1}^{j}(0)\right\rangle_{c}\right. & =\Lambda^{2}\left\langle\partial_{z} \xi^{i}(-i t) \partial_{z} \xi^{j}(0)\right\rangle \\
& =\Lambda^{2} \partial_{z} \partial_{z^{\prime}} G\left(t, t^{\prime} ; z, z^{\prime}\right)_{t^{\prime}=0, z=z^{\prime}=r / 2} \\
& =\tilde{\delta}^{i j} \frac{\pi \Lambda^{4}}{4 \sigma r^{2}} \sinh ^{-2}\left(\frac{\pi t}{2 r}\right)
\end{aligned}
$$

in which $\tilde{\delta}^{i j}=0$ for $i, j=3$, and $\tilde{\delta}^{i j}=\delta^{i j}$ for $i, j=1,2$. In a similar fashion, rest of the gauge field insertions are derived, from which the analytic expressions for the potentials are computed

$$
\begin{aligned}
& V^{(1,0)}(r)=\frac{g^{2} \Lambda^{4}}{2 \pi \sigma} \ln \left(\sigma r^{2}\right)+\mu_{1}, \quad V_{\mathbf{p}^{2}}^{(2,0)}(r)=0, \quad V_{\mathbf{L}^{2}}^{(2,0)}(r)=-\frac{g^{2} \Lambda^{4} r}{6 \sigma}, \quad V_{L S}^{(2,0)}(r)=-\frac{\mu_{2}}{r}-\frac{c_{F}^{(1)} g^{2} \Lambda^{2} \Lambda^{\prime}}{\sigma r^{2}} \\
& V_{r}^{(2,0)}(r)=-\frac{2 \zeta_{3} g^{4} \Lambda^{8} r}{\pi^{3} \sigma^{2}}+\mu_{3}+\frac{\mu_{4}}{r^{2}}+\frac{\mu_{5}}{r^{4}}+\frac{\pi^{3} c_{F}^{(1) 2} g^{2} \Lambda^{\prime \prime \prime 2}}{60 \sigma^{2} r^{5}}+\ldots, \quad \text { plus } V^{(1,1)}(r) \text { terms }
\end{aligned}
$$

where $\mu_{i}$, for $i \in\{1,2, \ldots, 5\}$, are the renormalization parameters, and $\zeta_{3}$ is the Riemann zeta function of argument three. Note that there arise a few (dimensionful) parameters, which are either to be determined or constrained by comparing to the lattice calculations. In order to simplify the task, however, one can exploit the symmetry of its underlying theory, Poincaré invariance in pNRQCD especially, which relates several potential terms, thereby the number of the parameters from the EST can be reduced. It is apparent that QCD is the Poincare invariant quantum field theory, and its lowenergy EFTs, NRQCD and pNRQCD in this context, also realize this symmetry as one truncates the action, order by order in the expansion parameters, and "impose" it to be invariant under the transformation; since the transformation is non-relativistic, only the spatial boost becomes non-trivial. 
By the invariance of the action up to total derivatives, some of the Wilson coefficients in front of the effective operators are related, and the relations between potentials from the pNRQCD Lagrangian are derived in this fashion. For instance, a relation between the static potential and the spin-orbit part of the potentials (the so-called Gromes relation [12]) constrains a renormalization parameter $\frac{1}{2 r} \frac{d V^{(0)}}{d r}+V_{L S}^{(2,0)}-V_{L_{2} S_{1}}^{(1,1)}=0 \longrightarrow \mu_{2}=\frac{\sigma}{2}$. The relation between momentum dependent potentials constrains the dimensionful parameter from the EST as well [13]: $\frac{r}{2} \frac{d V^{(0)}}{d r}+2 V_{\mathbf{L}^{2}}^{(2,0)}-V_{\mathbf{L}^{2}}^{(1,1)}=0 \longrightarrow$ $g \Lambda^{2}=\sigma$. Imposing all of these constraints to the parameters, the "finalized" potentials at the leading order are derived

$$
\begin{aligned}
& V^{(1,0)}(r)=\frac{\sigma}{2 \pi} \ln \left(\sigma r^{2}\right)+\mu_{1} \\
& V_{\mathbf{p}^{2}}^{(2,0)}(r)=0, \quad V_{\mathbf{L}^{2}}^{(2,0)}(r)=-\frac{\sigma r}{6}, \quad V_{L S}^{(2,0)}(r)=-\frac{\sigma}{2 r}-\frac{c_{F}^{(1)} g \Lambda^{\prime}}{r^{2}}, \quad V_{r}^{(2,0)}(r)=-\frac{2 \zeta_{3} \sigma^{2} r}{\pi^{3}} \\
& V_{\mathbf{p}^{2}}^{(1,1)}(r)=0, \quad V_{\mathbf{L}^{2}}^{(1,1)}(r)=\frac{\sigma r}{6}, \quad V_{L_{2} S_{1}}^{(1,1)}(r)=-\frac{c_{F}^{(1)} g \Lambda^{\prime}}{r^{2}}, \\
& V_{S^{2}}^{(1,1)}(r)=\frac{2 \pi^{3} c_{F}^{(1)} c_{F}^{(2)} g^{2} \Lambda^{\prime \prime \prime 2}}{45 \sigma^{2} r^{5}}, \quad V_{\mathbf{S}_{12}}^{(1,1)}(r)=\frac{\pi^{3} c_{F}^{(1)} c_{F}^{(2)} g^{2} \Lambda^{\prime \prime \prime 2}}{90 \sigma^{2} r^{5}}, \quad V_{r}^{(1,1)}(r)=-\frac{\zeta_{3} \sigma^{2} r}{2 \pi^{3}}
\end{aligned}
$$

and for the Hamiltonian of the system in the center of mass frame, $H=\mathbf{p}^{2} / M+V\left(M_{1,2}=M\right)$, the potential is summarized up to

$$
V(r) \approx \sigma r+\frac{1}{M} \frac{\sigma}{\pi} \ln \left(\sigma r^{2}\right)+\frac{1}{M^{2}}\left(-\frac{\sigma}{6 r} \mathbf{L}^{2}-\frac{\sigma}{2 r} \mathbf{L} \cdot \mathbf{S}-\frac{9 \zeta_{3} \sigma^{2} r}{2 \pi^{3}}\right)
$$

Significance of this result is the fact that the potential depends only on the two parameters: the string tension $\sigma$ and the heavy quark mass $M$, and these are to be compared to the lattice calculation.

\section{NLO to the heavy quarks potentials via the EST}

So far, the static singlet potential has been derived only up to the leading order in the EST. However, it is necessary for us to consider the next-to-leading (NLO) order in this EFT as those contributions can not only alter some coefficients of the leading order potentials, such as $V_{S^{2}}$ or $V_{r}$, but also some non-trivial terms arise like in the case of $V_{p^{2}}$. There are two different contributions to the NLO calculations in the EST. The first one is the leading order mapping but with including up to the NLO terms in the EST action, and the second is the NLO mapping from QCD to the EST with only including up to the leading order part of the action. According to the open-closed string duality at higher orders [14], the NLO terms are uniquely determined as $\left(\partial_{a} \xi^{l} \partial^{a} \xi^{l}\right)^{2},\left(\partial_{a} \xi^{l} \partial_{b} \xi^{l}\right)\left(\partial^{a} \xi^{m} \partial^{b} \xi^{m}\right)$. Thus, one has to derive the Green's function up to NLO via the perturbative expansion, and the emergence of tadpoles are to be treated by a regulaziation/renormalization scheme. 
As for the NLO contribution in the mapping, it is again uniquely determined by the symmetries ( $C P, T$, rotation, reflection) of the system as was used for the leading order mapping

$$
\begin{aligned}
\left.\left\langle\ldots \mathbf{E}_{1,2}^{l}(t) \ldots\right\rangle\right\rangle= & \left\langle\ldots \Lambda^{2} \partial_{z} \xi^{l}(t, \pm r / 2)+\bar{\Lambda}^{2} \partial_{z} \xi^{l}(t, \pm r / 2)\left(\partial_{a} \xi^{m}(t, \pm r / 2)\right)^{2} \ldots\right\rangle \\
\left\langle\left\langle\ldots \mathbf{B}_{1,2}^{l}(t) \ldots\right\rangle\right\rangle= & \left\langle\ldots \pm \Lambda^{\prime 2} \epsilon^{l m} \partial_{t} \partial_{z} \xi^{m}(t, \pm r / 2) \pm{\overline{\Lambda^{\prime}}}^{2} \epsilon^{l m} \partial_{t} \partial_{z} \xi^{m}(t, \pm r / 2)\left(\partial_{a} \xi^{n}(t, \pm r / 2)\right)^{2} \ldots\right\rangle \\
\left\langle\left\langle\ldots \mathbf{E}_{1,2}^{3}(t) \ldots\right\rangle\right\rangle= & \left\langle\ldots \Lambda^{\prime \prime 2}+{\overline{\Lambda^{\prime \prime}}}^{2}\left(\partial_{a} \xi^{m}(t, \pm r / 2)\right)^{2} \ldots\right\rangle \\
\left\langle\left\langle\ldots \mathbf{B}_{1,2}^{3}(t) \ldots\right\rangle\right\rangle= & \left\langle\cdots \pm \Lambda^{\prime \prime \prime} \epsilon^{l m} \partial_{t} \partial_{z} \xi^{l}(t, \pm r / 2) \partial_{z} \xi^{m}(t, \pm r / 2)\right. \\
& \left. \pm{\overline{\Lambda^{\prime \prime \prime}}}^{l m} \partial_{t} \partial_{z} \xi^{l}(t, \pm r / 2) \partial_{z} \xi^{m}(t, \pm r / 2)\left(\partial_{a} \xi^{n}(t, \pm r / 2)\right)^{2} \ldots\right\rangle
\end{aligned}
$$

in which $\bar{\Lambda}, \overline{\Lambda^{\prime}}, \overline{\Lambda^{\prime \prime}}$, and $\overline{\Lambda^{\prime \prime \prime}}$ are the dimensionful paramters emerging from the NLO mapping, which are to be smaller than $\Lambda, \Lambda^{\prime}, \Lambda^{\prime \prime}$, and $\Lambda^{\prime \prime \prime}$, respectively. It is clear that the NLO mapping is suppressed by a factor of $\left(r \Lambda_{Q C D}\right)^{-2}$, and there arise tadpoles from the string equivalence to the multiple gauge fields insertions, which are to be regulaized and renormalized. As for the simplicity of the argument here, let us limit ourselves on calculating the NLO contribution to the momentum dependent potentials like $V_{\mathbf{p}^{2}}^{(2,0)}, V_{\mathbf{p}^{2}}^{(1,1)}$, for which the following gauge field insertions are computed

$$
\begin{aligned}
\left\langle\left\langle\mathbf{E}_{1}^{3}(t) \mathbf{E}_{1,2}^{3}(0)\right\rangle\right\rangle_{c}^{N L O} & \equiv\left\langle\left\langle\mathbf{E}_{1}^{3}(t) \mathbf{E}_{1,2}^{3}(0)\right\rangle\right\rangle^{N L O}-\left[\left\langle\left\langle\mathbf{E}_{1}^{3}(t)\right\rangle{ }^{N L O}\left\langle\left\langle\mathbf{E}_{1,2}^{3}(0)\right\rangle\right\rangle^{L O}+\left\langle\left\langle\mathbf{E}_{1}^{3}(t)\right\rangle\right\rangle^{L O}\left\langle\left\langle\mathbf{E}_{1,2}^{3}(0)\right\rangle\right\rangle^{N L O}\right]\right. \\
& =\frac{{\overline{\Lambda^{\prime \prime}}}^{2} \pi^{2}}{\sigma^{2} r^{4}}\left[\cosh \left(\frac{\pi t}{r}\right) \mp 1\right]^{-2}
\end{aligned}
$$

so that the potentials are derived

$$
\left.V_{\mathbf{p}^{2}}^{(2,0)}\right|^{N L O}=\left[\frac{1}{3 \pi}+\frac{\pi}{9}\right] \frac{g^{2} \overline{\Lambda^{\prime \prime}}}{\sigma^{2} r}-\mu_{p^{2}}^{(2,0)},\left.\quad V_{\mathbf{p}^{2}}^{(1,1)}\right|^{N L O}=\left[\frac{2}{3 \pi}-\frac{\pi}{9}\right] \frac{g^{2} \overline{\Lambda^{\prime \prime}}}{\sigma^{2} r},
$$

for which $\overline{\Lambda^{\prime \prime}}<\Lambda^{\prime \prime}$ and $\mu_{p^{2}}^{(2,0)}$ is a renormalization parameter. These potentials are related to the static potential via Poincaré invariance $-4 V_{\mathbf{p}^{2}}^{(2,0)}+2 V_{\mathbf{p}^{2}}^{(1,1)}-V^{(0)}+r \frac{d V^{(0)}}{d r}=0$, where the static potential up to NLO is $V^{(0)}(r)=\sigma r+\mu-\frac{\pi}{12 r}$ [15]. Plugging all these expressions into the equation, we find two constraints

$$
4 \mu_{p^{2}}^{(2,0)}=\mu, \quad g{\overline{\Lambda^{\prime \prime}}}^{2}=\frac{\sigma}{2} .
$$

Note that these potentials are determined in terms of the fundamental parameters of the EST like $\mu$ and $\sigma$. Calculations for the rest of the potentials are performed in the similar fashion, whose results are to be shown in our upcoming publications.

\section{Summary and outlook}

In this presentation, we have briefly discussed about the historical development and the background to the QCD flux tube model, which originates from the Nambu's suggestion for the heavy mesons at the long-distance limit. Recent calculations on the correction terms to the static heavy quark potential shows the necessity to include the NLO contributions, for which two non-relativistic EFTs for QCD, NRQCD and pNRQCD, were briefly introduced. It was shown at the end of the introduction that Wilson loop expectation value from NRQCD and gauge fields insertions therein at large time limit are related to the heavy quark potentials in PNRQCD via matching calculations. On the other hand, 
based on the equivalence conjecture between the Wilson loop expectation at the large time limit and the partition function of the EST, we have shown how the heavy quark potentials at the long distance limit can be expressed in terms of the dynamics of the string field, and the corresponding potentials were computed at the leading order of the expansion parameter in the EST; in addition it was shown that the number of the dimensionful parameters arising from the EST can be reduced by exploiting the Poincare invariance in QCD. Then the NLO contributions to the potentials via the EST was schematically shown, from which simple but significant result for two of the momentum-dependent potentials were derived by using the same symmetry argument. Complete NLO calculations for the potentials via the EST are in progress whose final result is to be compared to the lattice calculations, thereby the remaining free parameters are constrained. Lastly, we suggest this approach might shed new light on the research field like in AdS/QCD, but the elaborate ideas are to be explored in the near future.

\section{References}

[1] K. Wilson, Phys. Rev. D 10, 2445 (1974)

[2] Y. Nambu, Phys. Lett. B 80, 372 (1979)

[3] J. Kogut, G. Parisi, Phys. Rev. Lett. 47, 17 (1981)

[4] N. Brambilla, A. Pineda, J. Soto, A. Vairo, Phys. Rev. D 63, 014023 (2000)

[5] A. Pineda, A. Vairo, Phys. Rev. D 63, 054007 (2001)

[6] G. Perez-Nadal, J. Soto, Phys. Rev. D 79, 114002 (2009)

[7] N. Brambilla, M. Groher, H. E. Martinez, A. Vairo, Phys. Rev. D 90, 114032 (2014)

[8] W. E. Caswell, G. P. Lepage, Phys. Lett. B 167, 437-442 (1986)

[9] G. T. Bodwin, E. Braaten, G. P. Lepage, Phys. Rev. D 51, 1125 (1995)

[10] N. Brambilla, A. Pineda, J. Soto, A. Vairo, Nucl. Phys. B 566, 275-310 (2000)

[11] M. Lüscher, Nucl. Phys. B 180, 317 (1981)

[12] D. Gromes, Z. Phys. C 26, 401 (1984)

[13] N. Brambilla, D. Gromes, A. Vairo, Phys. Lett. B 576, 314-327 (2003)

[14] O. Aharony, Z. Komargodski, JHEP 05, 118 (2013)

[15] M. Lüscher, K. Symanzik, P. Weisz, Nucl. Phys. B 173, 365 (1980) 